\title{
Computational Study on the Comparative Differences in the Activity of Inhibitors of Human versus Rat Alpha-Glucosidase
}

\author{
Shinya Nakamura*, Kazuko Shimada, Genzoh Tanabe, Osamu Muraoka, Isao Nakanishi \\ Department of Pharmaceutical Sciences, Kindai University, Higashiosaka, Japan \\ Email: *nakas@phar.kindai.ac.jp
}

How to cite this paper: Nakamura, S., Shimada, K., Tanabe, G., Muraoka, O. and Nakanishi, I. (2017) Computational Study on the Comparative Differences in the Activity of Inhibitors of Human versus Rat Alpha-Glucosidase. Open Journal of Medicinal Chemistry, 7, 19-28. https://doi.org/10.4236/ojmc.2017.72002

Received: May 1, 2017

Accepted: June 12, 2017

Published: June 15, 2017

Copyright () 2017 by authors and Scientific Research Publishing Inc. This work is licensed under the Creative Commons Attribution International License (CC BY 4.0).

http://creativecommons.org/licenses/by/4.0/

\begin{abstract}
Differences between the inhibitory activities of specific compounds on analogous enzymes isolated from different animal species are one of the critical issues to evaluate when exploring structure-activity relationships. The activity of acarbose is about ten times stronger in rat than in human, and that of neosalacinol is similar in both species. Binding affinities of acarbose and neosalacinol to four catalytic domains of alpha-glucosidases in human and rat were compared to investigate the cause of activity differences among species. Species difference was brought about complicatedly by the balance of interaction with four domains, and the result was indicated that larger ligand would show larger species difference in activity.
\end{abstract}

\section{Keywords}

Homology Modeling, MM/PBSA, Alpha-Glucosidase, Salacinol, Acarbose, Species Difference

\section{Introduction}

The human intestinal alpha-glucosidases, maltase-glucoamylase (MGAM) and sucrase-isomaltase (SI), have two catalytic domains in the $\mathrm{N}$-terminal side (NtMGAM, NtSI) and the C-terminal side (CtMGAM, CtSI) [1] [2]. These four catalytic domains are homologous, and every domain catalyzes maltose hydrolysis at the alpha-1,4-glycosidic bond. In addition, NtSI and CtSI catalyze the hydrolysis of isomaltose and sucrose, respectively [3]. To elicit their antidiabetic activity, alpha-glucosidase inhibitors must bind to all the mentioned domains to prevent disaccharide hydrolysis. We have investigated the structure-activity relationships of the compounds (salacinol, kotalanol and neosalacinol) isolated from Salacia reticulata [4] [5] [6] [7], and have designed novel derivatives from the 
human target protein structure [8]. The inhibitory activities of these compounds were assayed using the enzymes isolated from rat intestine. When assaying the activity of compound, the activity may be affected by species difference. For instance, it is reported that compound with agonist activity changed to antagonist in different animal species [9]. These species differences of the activities of compound are very difficult problem in rational drug design. Indeed, acarbose (a known alpha-glucosidase inhibitor, Figure 1(a)) and neosalacinol (Figure 1(b)) showed a different pattern of inhibitory activity between the human and rat enzyme [10]. These species-based differences in the inhibitory activities of salacinol derivatives should be investigated to increase our understanding of the structure-activity relationship. In this study, the species difference with regard to the inhibitory activity of these compounds has been examined by a computational method.

\section{Materials and Methods}

\subsection{Homology Modeling}

Maltose hydrolysis by alpha-glucosidases take place in all four catalytic domains. Binding affinities of acarbose and neosalacinol must be calculated for all four domains of the enzymes from the two animal species. The crystal structures of three domains of the human enzyme (NtMGAM, CtMGAM, and NtSI) have been reported (PDBID: 3L4Z [11], 3TOP [12], and 3LPP [13], respectively), whereas that of CtSI and those of all four domains of the rat enzyme are unknown. Therefore, the structures of the domains, for which relevant data were not available, were predicted by implementing a homology modeling method. In such an approach, the three-dimensional (3D) structure of a target protein is constructed from the experimental 3D structure of a homologous protein used as a template. To obtain a reliable model structure, an amino acid sequence homology greater than $30 \%$ is required between the target and the template protein [14]. As the homologies between the modeled domains and their templates listed in Table 1 exceeded this threshold, reliable model structure would be expected.

As the model structure of human CtSI has been reported in a previous paper [15], models of the rat enzyme catalytic domains were constructed from the

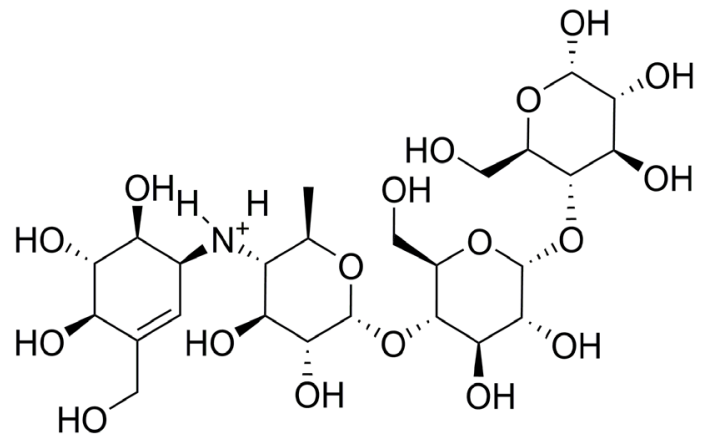

(a)<smiles>OC[C@H](O)C[S+]1CC(O)[C@@H](O)[C@H]1CO</smiles>

(b)

Figure 1. Compounds for comparison of binding affinities. (a) acarbose (b) neosalacinol. 
Table 1. Targetstructures and corresponding template structures in homology modeling.

\begin{tabular}{cccc}
\hline Target protein & Template protein & Template PDBID & Homology \\
\hline human CtSI & human CtMGAM & 3TOP & $60 \%$ \\
rat NtMGAM & human NtMGAM & $3 \mathrm{~L} 4 \mathrm{Z}$ & $57 \%$ \\
rat NtSI & human NtSI & $3 \mathrm{LPP}$ & $69 \%$ \\
rat CtMGAM & human CtMGAM & $3 \mathrm{TOP}$ & $76 \%$ \\
rat CtSI & human CtMGAM & 3TOP & $54 \%$ \\
\hline
\end{tabular}

known human domains in the same manner previously described [15]. The amino acid sequences of rat MGAM and rat SI were retrieved from NCBI-Gene ID: rno312272 and rno497756, respectively. After sequence alignment using the BLOSUM62 scoring matrix, all 3D structures were modeled by DS-MODELLER [16]. All amino acid protonation states and the positions of the hydrogen atoms were assigned by the Protonate-3D method [17] implemented in MOE [18].

\subsection{Binding Affinity Prediction}

Binding mode of the inhibitor is a critical factor in the prediction of the binding affinity. Each structure of acarbose and neosalacinol bound to the catalytic domain was constructed by superposition of the following crystal structures: acarbose with NtMGAM (PDBID: 2QMJ [19]) and salacinol with NtMGAM (PDBID $3 \mathrm{~L} 4 \mathrm{Z}$ [11]). After the ligands were placed in each domain, the sulfate group of salacinol was replaced by a hydroxy group to obtain the chemical structure of neosalacinol. Binding affinity between each ligand and enzyme domain was calculated by the MM-PBSA method [20].

Molecular dynamics (MD) simulations for 16 complexes (4 domains, 2 ligands, and 2 species) were performed using the AMBER software package [21]. Before the MD simulation, water molecules around each ligand located in the enzyme's binding pocket were generated to detect hydrogen-bond bridging water molecules by MOE-Solvent Analysis. Since alpha-glucosidases are very large proteins (about 900 amino acid residues and 13,000 atoms), to reduce computational cost, the binding pockets were capped by water molecules within a radius of $30 \AA$, and only the solvated region was kept movable during MD simulations. The AMBER ff99SB parameters [22] and GAFF/RESP parameters [23] [24] were assigned for protein and ligand atoms, respectively. A three-step energy minimization were performed: positions of hydrogen atoms, side chain atoms, and finally, all atoms were gradually optimized by implementing the steepest descent method and the conjugate gradient method for each 500 cycle step. After energy minimization and 500 ps heating, the system was equilibrated for 500 ps before data collection. Subsequently, an MD simulation of $2 \mathrm{~ns}$ was performed and the complex structures were extracted through 400 snapshots taken every 5 ps. This MD simulation scheme was replicated three times, and the binding affinities were calculated for a total of 1200 snapshots using the AMBER-MMPBSA.py module. [25]. At this point, the binding entropy term was calculated for 120 snapshots obtained at regular intervals from the 1200 snapshots by normal mode 
analysis with GBHTC implicit solvent model [26] and with truncated complex structure within $12 \AA$ from the ligand [27] to reduce computational costs.

\section{Results and Discussion}

\subsection{Homology Modeling}

The structure model quality of the four rat catalytic domains was evaluated by QMEAN6 score [28]. The values of $0.63,0.54,0.66$ and 0.70 for NtMGAM, CtMGAM, NtSI and CtSI, respectively. These values were high enough to allow comparisons between binding pockets to be conducted. All human and rat domains were superimposed on each other based on the protein main chain atoms and compared (Figure 2). The residues involved in hydrogen bonds and ionic

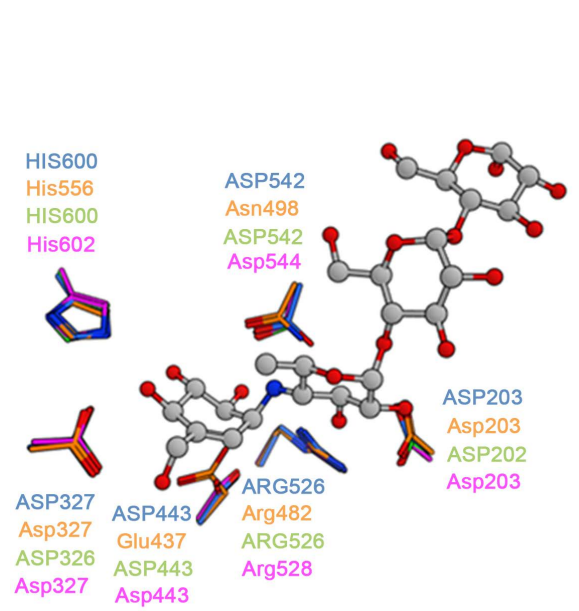

(a)

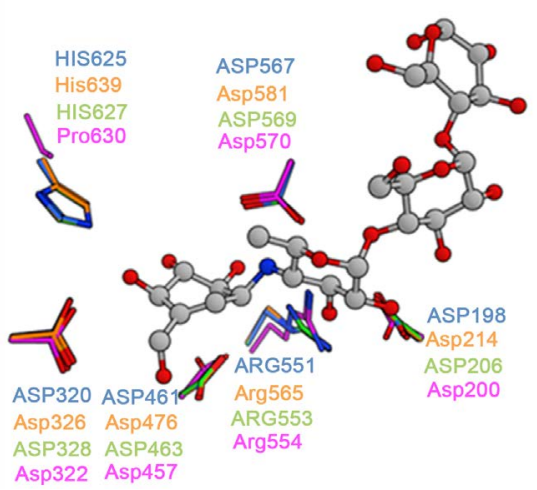

(c)

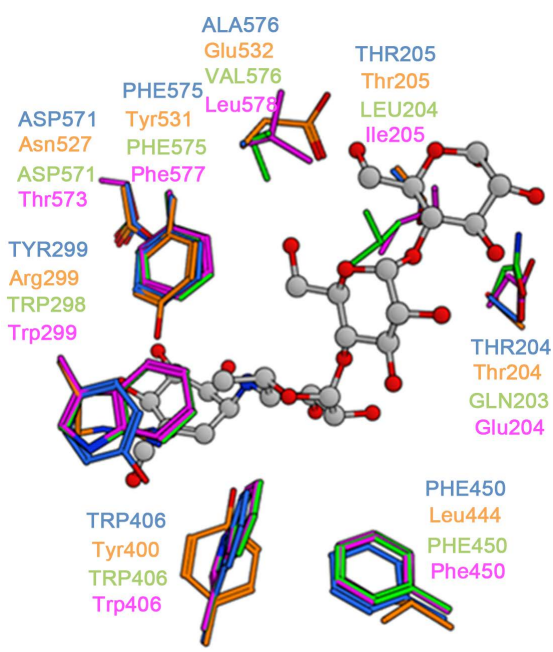

(b)

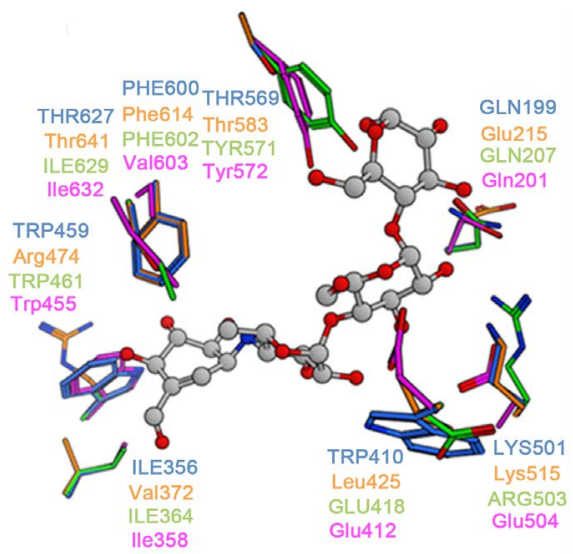

(d)

Figure 2. Comparisons of the ligand binding pockets with superposed acarbose. Acarbose is shown as a ball \& stick model. Blue: human MGAM, Orange: rat MGAM, Green: Human SI, Magenta: rat SI, (a) Residues of the N-terminal catalytic domains involved in hydrogen bonding; (b) non-conserved residues in the ligand-binding pocket of the N-terminal catalytic domains; (c) residues of the C-terminal catalytic domains involved in hydrogen bonding; (d) non-conserved residues in the ligand-binding pocket of the C-terminal catalytic domains. 
interactions were well conserved between human and rat domains except for Asn498/Glu437 in rat NtMGAM (Figure 2(a)) and Pro630 in rat CtSI (Figure $2(\mathrm{c}))$. Thus, polar interactions with the ligands should also be conserved between enzymes. However, we observed significant differences in terms of the identity of residues between human and rat domains. For the sake of clarity, in the rest of the text, residues of the human enzyme will be reported in all capital letters, whereas those of the rat enzyme will be reported using the regular notation. Hydrophobic TYR299 and ALA576 residues are found in the human NtMGAM domain, which correspond to hydrophilic residues Arg299 and Glu532, respectively, in the corresponding domain of the rat enzyme (Figure 2(b)). Likewise, TRP459 in human CtMGAM is replaced by Arg474 in the rat domain, the acidic ASP571 in human NtSI is replaced by the neutral Thr573 in rat NtSI (Figure 2(b)), and the basic ARG503 in human CtSI is replaced by the acidic Glu504 in rat CtSI (Figure 2(d)). Conserved residues are found at the bottom of the ligand-binding pockets, whereas mutated residues are located rather outside of the pocket. These differences are predicted to affect the inhibitory activity of large compounds such as acarbose.

\subsection{Binding Affinity Prediction}

Calculated binding affinities of the two compounds for each domain are reported in Table 2. Among the experimental inhibitory activities of alpha-glucosidase inhibitors, the strongest inhibition is observed for acarbose with respect to the rat enzyme. The calculated binding affinities averaged over four domains could not reproduce the experimental data on strongest inhibition; the calculated affinities of acarbose for alpha-glucosidases are both lower than those of neosalacinol. However, species-based differences clearly observed for each compound. These results may be affected by the estimated atomic partial charge of the compounds. As there are many charged residues around the ligand-binding pockets, affinity is sensitive to ligand partial charge. Although comparing the calculated affinities of two different ligands would, in this context, provide results that should be handled with care, comparing the binding affinities of a sin-

Table 2. Binding affinities $(\mathrm{kcal} / \mathrm{mol})$ for enzyme domain and enzyme inhibitory activity of two compounds.

\begin{tabular}{ccccc}
\hline & \multicolumn{2}{c}{ Acarbose } & \multicolumn{2}{c}{ Neosalacinol } \\
\cline { 2 - 5 } & Human & rat & Human & rat \\
\hline NtMGAM & -1.5 & -15.4 & -20.6 & -20.6 \\
NtSI & -4.3 & -2.0 & -12.5 & -27.6 \\
CtMGAM & -8.3 & -13.3 & -23.6 & -7.0 \\
CtSI & -12.5 & -12.4 & -14.1 & -13.9 \\
Average of four domains & -6.7 & -10.9 & -17.7 & -17.3 \\
IC $_{50}(\mu \mathrm{M})^{\text {a }}$ & 15.2 & 1.7 & 9.0 & 22.2 \\
\hline
\end{tabular}

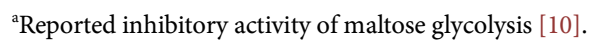


gle ligand for two different enzymes is reasonable. The experimental inhibitory activity of acarbose on alpha-glucosidase is about ten times lower for the human enzyme than for its rat counterpart, and that of neosalacinol is slightly higher for the human than for the rat enzyme. Furthermore, the binding affinities of acarbose for the human C-terminal domains of alpha-glucosidase are significantly higher than those calculated for the N-terminal domains. On the other hand, this difference was quite small in the case of neosalacinol. Although substrate specificities of the domains of the rat enzyme have not been investigated, the C-terminal domains of human alpha-glucosidase are known to be the site of hydrolysis of, not only disaccharides, but also tri-saccharides [29]; therefore, large ligands prefer $\mathrm{C}$-terminal domains over $\mathrm{N}$-terminal domains. The chemical size of acarbose corresponds to that of large tetra-saccharides, whereas the size of neosalacinol corresponds to that of small disaccharides. The binding affinities of each compound to the human domains reflected ligand-size and substrate specificity. Species-based differences in terms of inhibitory activity of acarbose are hypothesized to originate from the binding affinity for NtMGAM. Given the small size of its binding pocket, human NtMGAM prefers small ligands [15], so the large acarbose could not interact well with human NtMGAM. Consequently, acarbose inhibitory activity is lower for the human enzyme than for its rat counterpart. Almost half of the acarbose structure did not interact with NtMGAM (Figure 2(a)), and the inhibitor was exposed to the solvent in the crystal structure of the ligand-domain complex obtained by X-ray crystallography (PDBID: $2 \mathrm{QMJ})$. The binding affinities of neosalacinol for rat CtMGAM is lower than that for rat NtSI because of the interaction between the cationic neosalacinol and the charged residues; the neutral residue TRP549 in human CtMGAM corresponds to the cationic residue Arg474 in the rat enzyme, with cation-cation unfavorable interactions, and the neutral GLN203/ALA576 residues in human NtSI correspond to the anionic Glu204/Gly532 in rat NtSI with cation-anion favorable interactions. These characteristics would result in a slight species-based difference in the inhibitory activity of neosalacinol. In the prospect of future structure-based drug design efforts to identify suitable neosalacinol derivatives, we should keep in mind that is very complicated to consider all four domains. However, designing such drugs on the basis of the CtSI structure would be a simple and efficient approach to determine the substituent effects, as the relevant binding affinities between ligand and enzymes from the two different animal species are similar.

\section{Conclusions}

Two alpha-glucosidase inhibitors, acarbose and neosalacinol, have been reported to display different inhibitory activity toward the human-isolated versus the rat-isolated enzyme. In this study, the binding affinities of these compounds to four catalytic domains of the human and rat alpha-glucosidases have been evaluated computationally. The crystal structures of human NtMGAM, NtSI, and CtMGAM domains have been reported but those of the other domains have not; 
therefore, homology modeling of the unknown structures has been performed, and the ligand-binding pockets of all domains have been compared. The liganddomain complex structures were obtained by superimposing and modeling known complexes, and binding affinities calculated by the MM-PBSA method were then compared.

Averaged binding affinities of the compounds for four domains matched the experimental species differences in terms of inhibitory activity. The low inhibitory activity of acarbose with respect to the human enzyme is caused by the weak interaction of the inhibitor with the NtMGAM domain, which prefers ligands that are smaller than acarbose. The fact that the inhibitory effect of neosalacinol on the human enzyme is comparable to that observed for the rat enzyme is explained by a compensation of the charge interactions between rat NtSI and human CtMGAM. Namely, since maltose glycolysis takes place in four catalytic domains, the inhibitory activity of ligands is affected in a complex way by the interactions of the ligand with these four domains. Small ligands, whose size corresponded to that of disaccharides, are not very affected by structural differences among domains because these ligands occupy the catalytic core region, wherein disaccharide glycolysis occurs. However, large ligands could not settle stably in small pockets, and species-based differences in inhibitory activity would thus be enhanced. In this context, enlarging ligands by design and synthesis would probably not increase their activity. Activities predicted on the basis of calculated binding affinities of recently developed neosalacinol derivatives with large substituents have been estranged from the experimental result [30]. These results encouraged us to perform bioassays using human enzyme [31]. However, to improve the prediction accuracy of the inhibitory activities of designed compounds, it is necessary to refine further the structure of all domains, including with respect to features such as protonation state of pocket residues and ligands and the ratio of MGAM to SI in the human small intestine.

\section{References}

[1] Gray, G.M., Lally, B.C. and Conklin, K.A. (1979) Action of Intestinal SucraseIsomaltase and Its Free Monomers on an $\alpha$-Limit Dextrin. The Journal of Biological Chemistry, 254, 6038-6043. http://www.jbc.org/content/254/13/6038.citation

[2] Ernst, H.A., Leggio, L.L., Willemoës, M., Leonard, G., Blum, P. and Larsen, S. (2006) Structure of the Sulfolobus Solfataricus Alpha-Glucosidase: Implications for Domain Conservation and Substrate Recognition in GH31. Journal of Molecular Biology, 358, 1106-1124. https://doi.org/10.1016/j.jmb.2006.02.056

[3] Ren, L., Cao, X., Geng, P., Bai, F. and Bai, G. (2011) Study of the Inhibition of Two Human Maltase-Glucoamylases Catalytic Domains by Different $\alpha$-Glucosidase Inhibitors. Carbohydrate Research, 346, 2688-2692. https://doi.org/10.1016/j.carres.2011.09.012

[4] Tanabe, G., Yoshikai, K., Hatanaka, T., Yamamoto, M., Shao, Y., Minematsu, T., Muraoka, O., Wang, T., Matsuda, H. and Yoshikawa, M. (2007) Biological Evaluation of De-O-Sulfonated Analogs of Salacinol, the Role of Sulfate Anion in the Side Chain on the $\alpha$-Glucosidase Inhibitory Activity. Bioorganic \& Medicinal Chemistry, 15, 3926-3937. https://doi.org/10.1016/j.bmc.2006.10.014 
[5] Tanabe, G., Xie, W., Ogawa, A., Cao, C., Minematsu, T., Yoshikawa, M. and Muraoka, O. (2009) Facile Synthesis of De-O-Sulfated Salacinols: Revision of the Structure of Neosalacinol, a Potent $\alpha$-Glucosidase Inhibitor. Bioorganic \& Medicinal Chemistry Letters, 19, 2195-2198. https://doi.org/10.1016/j.bmcl.2009.02.103

[6] Eskandari, R., Jones, K., Rose, D.R. and Pinto, B.M. (2010) Probing the Active-Site Requirements of Human Intestinal N-Terminal Maltase Glucoamylase: The Effect of Replacing the Sulfate Moiety by a Methyl Ether in Ponkoranol, a Naturally Occurring $\alpha$-Glucosidase Inhibitor. Bioorganic \& Medicinal Chemistry Letters, 20, 5686-5689. https://doi.org/10.1016/j.bmcl.2010.08.020

[7] Xie, W., Tanabe, G., Akaki, J., Morikawa, T., Ninomiya, K., Minematsu, T., Yoshikawa, M., Wu, X. and Muraoka, O. (2011) Isolation, Structure Identification and SAR Studies on Thiosugar Sulfonium Salts, Neosalaprinol and Neoponkoranol, as Potent $\alpha$-Glucosidase Inhibitors. Bioorganic \& Medicinal Chemistry, 19, 2015-2022. https://doi.org/10.1016/j.bmc.2011.01.052

[8] Nakamura, S., Takahira, K., Tanabe, G., Morikawa, T., Sakano, M., Ninomiya, K., Yoshikawa, M., Muraoka, O. and Nakanishi, I. (2010) Docking and SAR Studies of Salacinol Derivatives as $\alpha$-Glucosidase Inhibitors. Bioorganic \& Medicinal Chemistry Letters, 20, 4420-4423. https://doi.org/10.1016/j.bmcl.2010.06.059

[9] Mowbray, C.E., Bell, A.S., Clarke, N.P., Collins, M., Jones, R.M., Lane, C.A., Liu, W.L., Newman, S.D., Paradowski, M., Schenck, E.J., Selby, M.D., Swain, N.A. and Williams, D.H. (2011) Challenges of Drug Discovery in Novel Target Space. The Discovery and Evaluation of PF-3893787: A Novel Histamine H4 Receptor Antagonist. Bioorganic \& Medicinal Chemistry Letters, 21, 6596-6602. https://doi.org/10.1016/j.bmcl.2011.07.125

[10] Morikawa, T., Miyake, S., Akaki, J., Ninomiya, K., Yoshikawa, M. and Muraoka, O. (2012) Quantitative analysis of Thiosugar Sulfoniums, Potent Alpha-Glucosidase Inhibitors from Salacia Species, Using LC-MS. 26th International Carbohydrate Symposium (ICS26).

[11] Chiasson, J.L., Josse, R.G., Gomis, R., Hanefeld, M., Karasik, A. and Laakso, M. (2002) Acarbose for Prevention of Type 2 Diabetes Mellitus: The STOP-NIDDM Randomised Trial. The Lancet, 359, 2072-2077. https://doi.org/10.1016/S0140-6736(02)08905-5

[12] Ren, L., Qin, X., Cao, X., Wang, L., Bai, F., Bai, G. and Shen, Y. (2011) Structural Insight into Substrate Specificity of Human Intestinal Maltase-Glucoamylase. Protein \& Cell, 2, 827-836. https://doi.org/10.1007/s13238-011-1105-3

[13] Sim, L., Willesma, C., Mohan, S., Naim, Y.H., Pinto, B.M. and Rose, D.R. (2010) Structural Basis for Substrate Selectivity in Human Maltase-Glucoamylase and Sucrase-Isomaltase N-Terminal Domains. Journal of Biological Chemistry, 285, 17763-17770. https://doi.org/10.1074/jbc.M109.078980

[14] Eswar, N., Webb, B., Marti-Renom, M.A., Madhusudhan, M.S., Eramian, D., Shen, M., Pieper, U. and Šali, A. (2006) Unit 5.6. Comparative Protein Structure Modeling Using Modeller. In: Current Protocols in Bioinformatics, Chapter 5, Wiley, Hoboken. https://doi.org/10.1002/0471250953.bi0506s15

[15] Nakamura, S., Takahira, K., Tanabe, G., Muraoka, O. and Nakanishi, I. (2012) Homology Modeling of Human Alpha-Glucosidase Catalytic Domains and SAR Study of Salacinol Derivatives. Open Journal of Medicinal Chemistry, 2, 50-60. https://doi.org/10.4236/ojmc.2012.23007

[16] Šali, A. and Blundell, T.L. (1993) Comparative Protein Modelling by Satisfaction of Spatial Restraints. Journal of Molecular Biology, 234, 779-815.

https://doi.org/10.1006/jmbi.1993.1626 
[17] Labute, P. (2009) Protonate3D: Assignment of Ionization States and Hydrogen Coordinates to Macromolecular Structures. Proteins, 75, 187-205. https://doi.org/10.1002/prot.22234

[18] MOE Ver (2013) Chemical Computing Group Inc., Montreal, Canada. http://www. chemcomp. com/

[19] Sim, L., Quezada-Calvillo, R., Sterchi, E.E., Nichols, B.L. and Rose, D.R. (2008) Human Intestinal Maltase-Glucoamylase: Crystal Structure of the N-Terminal Catalytic Subunit and Basis of Inhibition and Substrate Specificity. Journal of Molecular Biology, 375, 782-792. https://doi.org/10.1016/j.jmb.2007.10.069

[20] Kollman, P.A., Massova, I., Reyes, C., Kuhn, B., Huo, S., Chong, L., Lee, M., Lee, T., Duan, Y., Wang, W., Donini, O., Cieplak, P., Srinivasan, J., Case, D.A. and Cheatham III, T.E. (2000) Calculating Structures and Free Energies of Complex Molecules: Combining Molecular Mechanics and Continuum Models. Accounts of Chemical Research, 33, 889-897. https://doi.org/10.1021/ar000033j

[21] Pearlman, D.A., Case, D.A., Caldwell, J.W., Ross, W.S., Cheatham III, T.E., DeBolt, S., Ferguson, D., Seibel, G. and Kollman, P. (1995) AMBER, a Package of Computer Programs for Applying Molecular Mechanics, Normal Mode Analysis, Molecular Dynamics and Free Energy Calculations to Simulate the Structural and Energetic Properties of Molecules. Computer Physics Communications, 91, 1-41.

https://doi.org/10.1016/0010-4655(95)00041-d

[22] Hornak, V., Abel, R., Okur, A., Strockbine, B., Roitberg, A. and Simmerling, C. (2006) Comparison of Multiple Amber Force Fields and Development of Improved Protein Backbone Parameters. Proteins, 65, 712-725. https://doi.org/10.1002/prot.21123

[23] Wang, J., Wolf, R.M., Caldwell, J.W., Kollman, P.A. and Case III, D.A. (2004) Development and Testing of a General Amber Force Field. Journal of Computational Physics, 25, 1157-1174.

[24] Christopher, I.B., Piotr, C., Wendy, C. and Peter, A.K. (1993) A Well-Behaved Electro-Static Potential Based Method Using Charge Restraints for Deriving Atomic Charges: The RESP Model. The Journal of Physical Chemistry, 97, 10269-10280. https://doi.org/10.1021/j100142a004

[25] Miller III, B.R., McGee Jr., T.D., Swails, J.M., Homeyer, N., Gohlke, H. and Roitberg, A.E. (2012) MMPBSA.py: An Efficient Program for End-State Free Energy Calculations. Journal of Chemical Theory and Computation, 8, 3314-3321. https://doi.org/10.1021/ct300418h

[26] Hawkins, G.D., Cramer, C.J. and Truhlar, D.G. (1996) Parametrized Models of Aqueous Free Energies of Solvation Based on Pairwise Descreening of Solute Atomic Charges from a Dielectric Medium. The Journal of Physical Chemistry, 100, 19824-19839. https://doi.org/10.1021/jp961710n

[27] Kongsted, U. and Ryde, U. (2009) An Improved Method to Predict the Entropy Term with the MM/PBSA Approach. The Journal of Computer-Aided Molecular Design, 23, 63-71. https://doi.org/10.1007/s10822-008-9238-Z

[28] Benkert, P., Biasini, M. and Schwede, T. (2011) Toward the Estimation of the Absolute Quality of Individual Protein Structure Models. Bioinformatics, 27, 343-350. https://doi.org/10.1093/bioinformatics/btq662

[29] Jones, K., Sim, L., Mohan, S., Kumarasamy, J., Liu, H., Avery, S., Naim, H.Y., Quezada-Calvillo, R., Nichols, B.L., Pinto, B.M. and Rose, D.R. (2011) Mapping the Intestinal Alpha-Glucogenic Enzyme Specificities of Starch Digesting Maltase-Glucoamylase and Sucrase-Isomaltase. Bioorganic \& Medicinal Chemistry, 19, 39293934. https://doi.org/10.1016/j.bmc.2011.05.033 
[30] Tanabe, G., Nakamura, S., Tsutsui, N., Balakisian, G., Xie, W., Tsuchiya, S., Akaki, J., Morikawa, T., Ninomiya, K., Nakanishi, I., Yoshikawa, M. and Muraoka, O. (2012) In Silico Design, Synthesis and Evaluation of 3'-O-Benzylated Analogs of Salacinol, a Potent $\alpha$-Glucosidase Inhibitor Isolated from an Ayurvedic Traditional Medicine "Salacia". Chemical Communications, 48, 8646-8648. https://doi.org/10.1039/c2cc34144a

[31] Tanabe, G., Xie, W., Balakishan, G., Amer, M.F., Tsutsui, N., Takemura, H., Nakamura, S., Akaki, J., Ninomiya, K., Morikawa, T., Nakanishi, I. and Muraoka, O. (2016) Hydrophobic Substituents Increase the Potency of Salacinol, a Potent $\alpha$ Glucosidase Inhibitor from Ayurvedic Traditional Medicine "Salacia". Bioorganic \& Medicinal Chemistry, 24, 3705-3715. https://doi.org/10.1016/j.bmc.2016.06.013

Submit or recommend next manuscript to SCIRP and we will provide best service for you:

Accepting pre-submission inquiries through Email, Facebook, LinkedIn, Twitter, etc. A wide selection of journals (inclusive of 9 subjects, more than 200 journals)

Providing 24-hour high-quality service

User-friendly online submission system

Fair and swift peer-review system

Efficient typesetting and proofreading procedure

Display of the result of downloads and visits, as well as the number of cited articles

Maximum dissemination of your research work

Submit your manuscript at: http://papersubmission.scirp.org/

Or contact ojmc@scirp.org 